\title{
The effect of the Gauss-Bonnet term on Hawking radiation from arbitrary dimensional black brane
}

\author{
Xiao-Mei Kuang ${ }^{1,2, a}$, Joel Saavedra ${ }^{1, b}$, Ali Övgün ${ }^{1,3, c}$ \\ ${ }^{1}$ Instituto de Física, Pontificia Universidad Católica de Valparaíso, Casilla, 4950 Valparaíso, Chile \\ ${ }^{2}$ Center for Gravitation and Cosmology, College of Physical Science and Technology, Yangzhou University, Yangzhou 225009, China \\ ${ }^{3}$ Physics Department, Eastern Mediterranean University, Famagusta, Northern Cyprus
}

Received: 4 July 2017 / Accepted: 4 September 2017 / Published online: 15 September 2017

(C) The Author(s) 2017. This article is an open access publication

\begin{abstract}
We investigate the probabilities of the tunneling and the radiation spectra of massive spin-1 particles from arbitrary dimensional Gauss-Bonnet-Axions (GBA) Antide Sitter (AdS) black branes, via using the WKB approximation to the Proca spin-1 field equation. The tunneling probabilities and Hawking temperature of the arbitrary dimensional GBA AdS black brane is calculated via the HamiltonJacobi approach. We also compute the Hawking temperature via the Parikh-Wilczek tunneling approach. The results obtained from the two methods are consistent. In our setup, the Gauss-Bonnet (GB) coupling affects the Hawking temperature if and only if the momentum of the axion fields is non-vanishing.
\end{abstract}

\section{Introduction}

Since Hawking proposed that black holes are not actually black, inasmuch as they emit a nearly thermal radiation, and this radiation causes them to lose energy, shrink and eventually disappear. This is a disaster as regards the laws of quantum physics because information is lost, which is the unsolved black hole information problem [1,2]. Recently, Hawking, Perry and Strominger showed some ways to solve the information problem by defining new terms in physics: 'soft particles' [3,4] and 'hard particles' [5]. Soft particles carry information and imprint this information on the radiation, even after the black hole itself is defunct.

In the process of constructing quantum gravity theory, physicists keep a strong interests in the study of Hawking radiation proposed in $[1,2]$. In order to strengthen this staggering theory, in the last decades, plenty of methods have

\footnotetext{
a e-mail: xmeikuang@gmail.com

be-mail: joel.saavedra@pucv.cl

c e-mail: ali.ovgun@pucv.cl
}

been proposed to derive Hawking radiation and calculate its temperature [6-12]. Among them, the semi-classical quantum tunneling method proposed in [8] has attracted much attention and shown remarkable progress. The trick in the method is to consider the Hawking radiation as a tunneling process of particles from the event horizon, and the tunneling probability for the classically forbidden trajectory to outside of the horizon is $\Gamma=\mathrm{e}^{-\frac{2}{\hbar} \operatorname{Im} S}$. So the crucial task is to compute the imaginary part of the classical action $\operatorname{Im} S$.

In the tunneling method, there are two popular approaches to calculate $\operatorname{Im} S$. One is the Parikh-Wilczek approach, which applies the null geodesic equation of the emitted scalar particles [8]. This approach has been extensively used in [1315]. The other way is the Hamilton-Jacobi approach, which mainly utilizes the Hamilton-Jacobi equation of the classical scalar particles proposed in [16-18]. Later, tunneling of Dirac fermions [19-25] and tunneling of gravitinos [26,27] have been imposed to study the Hawking radiation. More recently, tunneling of massive spin-1 vector particles has been widely studied to probe the radiation, which was first researched in $[28,29]$. This kind of tunneling was studied via the WKB approximation of the Proca equation, which describes the dynamics of spin-1 vector particles. Tunneling of the different types of particles has been generalized to more objects; see for examples [30-78].

In this paper, we will explore the quantum tunneling process of massive spin-1 vector particles emitted from arbitrary dimensional AdS black branes with GB corrections as well as axion fields found in [79]. ${ }^{1}$ To calculate the tunneling probability and the Hawking temperature, we first use

\footnotetext{
${ }^{1}$ We note that the Hawking temperature might be obtained using the tunneling of scalar, vector or Dirac particles, and the result is independent of the spin type of the particles. In this paper, we will only consider the massive vector particles which should give the correct Hawking temperature, because the study of vector particles is more recent and physically reasonable.
} 
the Hamilton-Jacobi approach by starting with the Proca equation; then we verify our results via the Parikh-Wilczek approach.

The motivations of our study stem from the following three aspects. Firstly, the study of tunneling of spin- 1 particles is very significant in particle physics because a vector boson comes with spin- 1 and the massive vector bosons $W^{ \pm}$and $Z^{0}$ particles (force carriers of the weak interaction) play an indispensable role against the background of the confirmed Higgs boson [80,81]. Secondly, it is well known that the gravitational theories with higher curvature coupling, such as GB corrections and the higher dimensions are very important and contain much richer physics. Thirdly, from the point view of the application of anti-de Sitter/conformal field theory, massless axion fields break the translation symmetry of the dual boundary theory in a very simple way. This was first discussed in [82]; thus we witness the introduction of finite DC conductivity in the study of holographic applications.

As is well known, the temperature of GB black branes with planar spatial topology does not depend on the GB parameters [83-85]. However, in the GBA gravitational theory constructed in [79], via the Euclidean method, the authors found that the temperature of GB AdS planar black hole with axion fields is dependent of the GB coupling. When there is no axion field, the temperature recovers that in normal GB theory. Thus, the aim of this paper is to calculate the Hawking temperature via the tunneling method and further confirm the result obtained in [79].

The remaining of this paper is organized as follows. In Sect. 2, we will review the black brane solution in GaussBonnet-Axions theory with arbitrary dimensions. Using the Hamilton-Jacobi approach, we compute the tunneling probabilities of the spin-1 vector particles and Hawking temperature of the black brane in Sect. 3, and we verify the Hawking temperature with the use of the Parikh-Wilczek approach in Sect. 3. The last section is for our conclusion and a discussion.

\section{Review of black brane in Gauss-Bonnet-Axions theory with arbitrary dimensions}

In this section, we will review the black hole solutions in arbitrary dimensional Gauss-Bonnet-Axions (GBA) theory proposed in [79] with the action

$S=\frac{1}{2 \kappa^{2}} \int d^{d+2} x \sqrt{-g}\left(R-2 \Lambda+\frac{\alpha}{2} \mathcal{L}_{G B}-\frac{1}{2} \sum_{I=1}^{d}\left(\partial \psi_{I}\right)^{2}\right)$.

Here $2 \kappa^{2}=16 \pi G_{d+2}$ is the $d+2$ dimensional gravitational coupling constant and $\alpha$ is the GB coupling constant. $\psi_{I}$ are a set of axionic fields and $\Lambda=-d(d+1) / 2 L^{2}$ is the cosmological constant while
$\mathcal{L}_{G B}=\left(R_{\mu \nu \rho \sigma} R^{\mu \nu \rho \sigma}-4 R_{\mu \nu} R^{\mu \nu}+R^{2}\right)$.

In what follows, we shall set $L=1$.

Variating the action, we can obtain the equations of motion as

$$
\begin{aligned}
& \nabla_{\mu} \nabla^{\mu} \psi_{I}=0, \\
& R_{\mu \nu}-\frac{1}{2} g_{\mu \nu}(R+d(d+1) \\
& \left.+\frac{\alpha}{2}\left(R^{2}-4 R_{\rho \sigma} R^{\rho \sigma}+R_{\lambda \rho \sigma \tau} R^{\lambda \rho \sigma \tau}\right)\right) \\
& +\frac{\alpha}{2}\left(2 R R_{\mu \nu}-4 R_{\mu \rho} R_{\nu}{ }^{\rho}-4 R_{\mu \rho \nu \sigma} R^{\rho \sigma}+2 R_{\mu \rho \sigma \lambda} R_{\nu}{ }^{\rho \sigma \lambda}\right) \\
& -\sum_{I=1}^{d}\left(\frac{1}{2} \partial_{\mu} \psi_{I} \partial_{\nu} \psi_{I}-\frac{g_{\mu \nu}}{4}\left(\partial \psi_{I}\right)^{2}\right)=0 .
\end{aligned}
$$

We take the form of the scalar fields linearly depending on the $d$ spatial direction $x^{a}$ where $a=1,2, \ldots d$ as

$\psi_{I}=\beta \delta_{I a} x^{a}$,

which breaks the translational symmetry in the dual field theory in a simple way [82]. The equations of motions admit a homogeneous and isotropic neutral black brane solution with planar topology ${ }^{2}$

$\mathrm{d} s^{2}=-f(r) \mathrm{d} t^{2}+\frac{1}{f(r)} \mathrm{d} r^{2}+\frac{r^{2}}{L_{e}^{2}} \sum_{a=1}^{d} \mathrm{~d} x^{a} \mathrm{~d} x^{a}$,

where, defining $\hat{\alpha}=(d-1)(d-2) \alpha / 2$,

$$
f(r)=\frac{r^{2}}{2 \hat{\alpha}}\left(1-\sqrt{1-4 \hat{\alpha}\left(1-\frac{r_{h}^{d+1}}{r^{d+1}}\right)+\frac{2 \hat{\alpha}}{r^{2}} \frac{L_{e}^{2} \beta^{2}}{d-1}\left(1-\frac{r_{h}^{d-1}}{r^{d-1}}\right)}\right) .
$$

Here $r_{h}$ satisfying $f\left(r_{h}\right)=0$ is the black brane horizon.

The constraint of the GB coupling parameter $\hat{\alpha}$ has been widely studied in arbitrary dimensions. Considering holography, the physical range of $\hat{\alpha}$ can mainly be determined by two constraints. One constraint is to require no negative energy fluxes to appear in all tensor, vector and scalar channels of perturbations, which can be achieved by computing the energy flux via holography and then translate the constraints of no negative energy fluxes condition into the constraint on the GB parameter [87]. The other constraint is to demand that the dual conformal field theory of GB theory is causal. It means that the local "speed of graviton" should not be higher than the speed of light, which can be realized via analyzing the potentials of the channels of gravitational

\footnotetext{
${ }^{2}$ In order to have a unit velocity of light, the metric ansatz $g_{a a}$ are dependent of GB coupling parameter $\hat{\alpha}$. They are somewhat different from the one presented in [86] in five dimensions where $g_{a a}=r^{2} / L^{2}$ is independent of the GB coupling $\hat{\alpha}$.
} 
perturbations [88]. From [87-90], the above two constraints produced similar physical ranges of the GB coupling parameter, which is

$$
-\frac{(d-1)(3 d+5)}{4(d+3)^{2}} \leqslant \hat{\alpha} \leqslant \frac{(d-1)(d-2)\left((d-1)^{2}+3 d+5\right)}{4\left((d-1)^{2}+d+3\right)^{2}} .
$$

It is worthy to note that it was shown in [91] that the constraint in the higher derivative coupling coming from causality issues is much more severe, especially in a weakly coupled theory. Later in [92], the authors pointed out that the causality violations can be cured by considering the Regge behavior, and more recent studies of the (in)stability can be found in [93-95]. The preciser causality constraint of the higher correction coupling is worthy of further investigation. Moreover, the result is given in the case without the axion fields. It may become more complicated due to the introduction of axionic fields [96].

Near the UV boundary, $r \rightarrow \infty$,

$f(r) \sim \frac{1-\sqrt{1-4 \hat{\alpha}}}{2 \hat{\alpha}} r^{2}$.

So the effective asymptotic AdS radius is

$L_{\mathrm{e}}^{2}=\frac{2 \hat{\alpha}}{1-\sqrt{1-4 \hat{\alpha}}} \rightarrow \begin{cases}1, & \text { for } \hat{\alpha} \rightarrow 0, \\ \frac{1}{2}, & \text { for } \hat{\alpha} \rightarrow \frac{1}{4} .\end{cases}$

Then, using the Euclidean method, the Hawking temperature of the black brane is

$T=\frac{f^{\prime}\left(r_{h}\right)}{4 \pi}=\frac{1}{4 \pi}\left((d+1) r_{h}-\frac{L_{e}^{2} \beta^{2}}{2 r_{h}}\right)$,

depending on the GB coupling via $L_{e}$ when $\beta \neq 0$, which is very different from the GB gravitational case with $\beta=0$.

The Einstein limit of the black brane solution is obtained by taking $\hat{\alpha} \rightarrow 0$, in which the gravitational background recovers the solution addressed in [82] with the redshift

$f(r)=r^{2}\left(1-\frac{r_{h}^{d+1}}{r^{d+1}}\right)-\frac{\beta^{2}}{2(d-1)}\left(1-\frac{r_{h}^{d-1}}{r^{d-1}}\right)$

and the temperature

$T=\frac{f^{\prime}\left(r_{h}\right)}{4 \pi}=\frac{1}{4 \pi}\left((d+1) r_{h}-\frac{\beta^{2}}{2 r_{h}}\right)$.

It is worth to point out that the dimension of the solution (6) can be $d \geqslant 2$ for the Einstein case with $\hat{\alpha} \rightarrow 0$, while for the GB case with $\hat{\alpha} \neq 0$, we have $d \geqslant 3$ since the Gauss-Bonnet term is a topological invariant in four dimensions [84].
3 Quantum Tunneling of Spin-1 Particles from $d+2$ dimensional Black Brane in Gauss-Bonnet-Axions Theory

Our main aim of this section is to calculate the Hawking temperature using the semi-classical Hamilton-Jacobi approach with the suitable WKB ansatz from the recently found solution of the black brane in GBA theory. In order to calculate the Hawking temperature we consider the quantum mechanically tunneling massive spin- 1 particles from the black brane in the GBA theory. The wave of the massive spin- 1 field $\psi_{\nu}$ satisfies the dynamical Proca equation [28],

$\frac{1}{\sqrt{-g}} \partial_{\mu}\left(\sqrt{-g} \psi^{\nu \mu}\right)+\frac{m^{2}}{\hbar^{2}} \psi^{\nu}=0$

where $m$ is the mass of the spin- 1 particles and $\psi_{\mu \nu}$ is the second-rank tensor which is defined by $\psi_{\mu \nu}=\partial_{\mu} \psi_{\nu}-$ $\partial_{\nu} \psi_{\mu}$. Note that here $\mu$ and $\nu$ refer to the coordinates $t, r, x^{1}, x^{2} \ldots x^{d}$.

We expand the Proca equation in the background of the black brane (5) in the GBA theory and find that in arbitrary dimensions, $D=d+2$, we have the components of the equations

$$
\begin{aligned}
v= & t: \frac{m^{2}}{\hbar^{2} f} \psi_{t}+\frac{\mathrm{d}}{r}\left(\frac{\partial \psi_{t}}{\partial r}-\frac{\partial \psi_{r}}{\partial t}\right)+\frac{\partial^{2} \psi_{t}}{\partial r^{2}}-\frac{\partial^{2} \psi_{r}}{\partial t \partial r} \\
& +\frac{L_{\mathrm{e}}^{2}}{r^{2} f}\left(\sum_{a=1}^{d} \frac{\partial^{2} \psi_{t}}{\partial x^{a 2}}-\sum_{a=1}^{d} \frac{\partial^{2} \psi_{x^{a}}}{\partial t \partial x^{a}}\right)=0, \\
v= & r: \frac{m^{2} f}{\hbar^{2}} \psi_{r}+\frac{\partial^{2} \psi_{t}}{\partial t \partial r}-\frac{\partial^{2} \psi_{r}}{\partial t^{2}} \\
& +\frac{L_{\mathrm{e}}^{2} f}{r^{2}}\left(\sum_{a=1}^{d} \frac{\partial^{2} \psi_{r}}{\partial x^{a 2}}-\sum_{a=1}^{d} \frac{\partial^{2} \psi_{x^{a}}}{\partial r \partial x^{a}}\right)=0, \\
v= & x^{1}: \frac{m^{2} L_{\mathrm{e}}^{2}}{\hbar^{2} r^{2}} \psi_{x^{1}}+\frac{L_{\mathrm{e}}^{4}}{r^{4}}\left(\sum_{a=2}^{d} \frac{\partial^{2} \psi_{x^{1}}}{\partial x^{a 2}}-\sum_{a=2}^{d} \frac{\partial^{2} \psi_{x^{a}}}{\partial x^{1} \partial x^{a}}\right) \\
& +\frac{L_{\mathrm{e}}^{2}}{r^{2}}\left[\left(\frac{\partial \psi_{x^{1}}}{\partial r}-\frac{\partial \psi_{r}}{\partial x^{1}}\right)\left(f^{\prime}+\frac{(d-2) f}{r}\right)\right. \\
& \left.+f\left(\frac{\partial \psi_{x^{1}}^{2}}{\partial r^{2}}-\frac{\partial \psi_{r}^{2}}{\partial r \partial x^{1}}\right)+\frac{1}{f}\left(\frac{\partial^{2} \psi_{t}}{\partial t \partial x^{1}}-\frac{\partial^{2} \psi_{x^{1}}}{\partial t^{2}}\right)\right]=0, \\
v & x^{j}: \frac{m^{2} L_{\mathrm{e}}^{2}}{\hbar^{2} r^{2}} \psi_{x^{j}}+\frac{L_{\mathrm{e}}^{4}}{r^{4}}\left(\sum_{a=1, a \neq j}^{d} \frac{\partial^{2} \psi_{x^{j}}}{\partial x^{a 2}}-\sum_{a=1, a \neq j}^{d} \frac{\partial^{2} \psi_{x^{a}}}{\partial x^{j} \partial x^{a}}\right) \\
& +\frac{L_{\mathrm{e}}^{2}}{r^{2}}\left[\left(\frac{\partial \psi_{x^{j}}}{\partial r}-\frac{\partial \psi_{r}}{\partial x^{j}}\right)\left(f^{\prime}+\frac{(d-2) f}{r}\right)\right. \\
& \left.\left.+f\left(\frac{\partial \psi_{x^{j}}^{2}}{\partial r^{2}}-\frac{\partial \psi_{r}^{2}}{\partial r \partial x^{j}}\right)+\frac{1}{f}\left(\frac{\partial^{2} \psi_{t}}{\partial t \partial x^{j}}-\frac{\partial^{2} \psi_{x^{j}}}{\partial t^{2}}\right)\right]=0, \quad(14)\right]
\end{aligned}
$$

where the index $j=2,3 \ldots d$ and the prime denotes the derivative to the radial coordinate $r$. Then, using the 
Hamilton-Jacobi method, we choose a suitable ansatz of the spin-1 vector field, [29]

$$
\begin{aligned}
\psi_{\nu}=C_{\nu} \exp [ & \frac{i}{\hbar}\left(S_{0}\left(t, r, x^{1}, x^{2}, \ldots, x^{d}\right)\right) \\
& \left.+\hbar S_{1}\left(t, r, x^{1}, x^{2}, \ldots, x^{d}\right)+\cdots\right],
\end{aligned}
$$

where $C_{v}=\left(C_{t}, C_{r}, C_{x^{1}}, C_{x^{2}}, \ldots, C_{x^{d}}\right)$ are some constants. It is noted that a classical action of the massive spin-1 particles is defined by $S_{0}\left(t, r, x^{1}, x^{2}, \ldots, x^{d}\right)$, which is a kinetic term [97]. Higher order correction terms $S_{i=1,2, . .}\left(t, r, x^{1}, x^{2}, \ldots, x^{d}\right)$ can be ignored because the particles can be considered to be free and without selfinteraction. Moreover, to choose the suitable WKB ansatz, one should use the symmetry of the background spacetime by Killing vectors [18]. In our case, we choose the action to leading order as follows:

$S_{0}\left(t, r, x^{1}, x^{2}, \ldots\right)=-E t+R(r)+\sum_{a=1}^{d} X^{a}\left(x^{a}\right)+c$,

which means that the particles of massive spin-1 have an energy of $E$. Here $c$ is a complex constant. Then we substitute Eqs. (15) and (16) into the components of the Proca equation given in Eq. (14), the corresponding quadruple equations with the lowest order in $\hbar$ can be obtained:

$$
\begin{aligned}
& {\left[\frac{m^{2}}{f}+\left(\frac{\partial R}{\partial r}\right)^{2}-\frac{L_{\mathrm{e}}^{2}}{r^{2} f} \sum_{a=1}^{d}\left(\frac{\partial X^{a}}{\partial x^{a}}\right)^{2}\right] C_{t}} \\
& -E \frac{\partial R}{\partial r} C_{r}-\frac{L_{\mathrm{e}}^{2} E}{r^{2} f} \sum_{a=1}^{d}\left(\frac{\partial X^{a}}{\partial x^{a}} C_{x^{a}}\right)=0 \\
& E \frac{\partial R}{\partial r} C_{t}+\left[m^{2} f+E^{2}+\frac{L_{\mathrm{e}}^{2} f}{r^{2}} \sum_{a=1}^{d}\left(\frac{\partial X^{a}}{\partial x^{a}}\right)^{2}\right] C_{r} \\
& +\frac{L_{\mathrm{e}}^{2} f}{r^{2}} \frac{\partial R}{\partial r} \sum_{a=1}^{d}\left(\frac{\partial X^{a}}{\partial x^{a}} C_{x^{a}}\right)=0 \\
& \frac{L_{\mathrm{e}}^{2} E}{r^{2} f} \frac{\partial X^{1}}{\partial x^{1}} C_{t}+\frac{L_{\mathrm{e}}^{2} f}{r^{2}} \frac{\partial R}{\partial r} \frac{\partial X^{1}}{\partial x^{1}} C_{r}+\frac{L_{\mathrm{e}}^{4}}{r^{4}} \frac{\partial X^{1}}{\partial x^{1}} \sum_{a=2}^{d}\left(\frac{\partial X^{a}}{\partial x^{a}} C_{x^{a}}\right) \\
& +\left[\frac{m^{2} L_{\mathrm{e}}^{2}}{r^{2}}-\frac{L_{\mathrm{e}}^{4}}{r^{4}} \sum_{a=2}^{d}\left(\frac{\partial X^{a}}{\partial x^{a}}\right)^{2}+\frac{L_{\mathrm{e}}^{2}}{r^{2}}\left(\frac{E^{2}}{f}-f\left(\frac{\partial R}{\partial r}\right)^{2}\right)\right] C_{x^{1}}=0
\end{aligned}
$$

$$
\begin{aligned}
& \frac{L_{\mathrm{e}}^{2} E}{r^{2} f} \frac{\partial X^{j}}{\partial x^{j}} C_{t}+\frac{L_{\mathrm{e}}^{2} f}{r^{2}} \frac{\partial R}{\partial r} \frac{\partial X^{j}}{\partial x^{j}} C_{r}+\frac{L_{\mathrm{e}}^{4}}{r^{4}} \frac{\partial X^{j}}{\partial x^{j}} \sum_{a=1, a \neq j}^{d}\left(\frac{\partial X^{a}}{\partial x^{a}} C_{x^{a}}\right) \\
& +\left[\frac{m^{2} L_{\mathrm{e}}^{2}}{r^{2}}-\frac{L_{\mathrm{e}}^{4}}{r^{4}} \sum_{a=1, a \neq j}^{d}\left(\frac{\partial X^{a}}{\partial x^{a}}\right)^{2}+\frac{L_{\mathrm{e}}^{2}}{r^{2}}\left(\frac{E^{2}}{f}-f\left(\frac{\partial R}{\partial r}\right)^{2}\right)\right] C_{x^{j}}=0,
\end{aligned}
$$

where again $j=2,3 \ldots d$.
We will use the matrix formalism to solve these $d+$ 2 equations for the radial function of $R(r)$. Let us first rewrite Eq. $(17)$ in a $(d+2) \times(d+2)$ matrix form as $\Lambda_{(d+2) \times(d+2)}\left(C_{t}, C_{r}, C_{x^{1}} \ldots C_{x^{d}}\right)^{\mathcal{T}}=0$, where $\mathcal{T}$ is the transition to the transposed vector. It is notable that here $\Lambda_{(d+2) \times(d+2)}$ is in matrix form and the function of the coordinates, and indeed $C_{\mu}$ are constants defined in Eq. (15). So we can read off all the elements of the matrix $\Lambda_{(d+2) \times(d+2)}$ from the related equations $(17 \mathrm{a})-(17 \mathrm{~d})$, thus:

$$
\begin{aligned}
& \Lambda_{1,1}=\frac{m^{2}}{f}+\left(\frac{\partial R}{\partial r}\right)^{2}-\frac{L_{\mathrm{e}}^{2}}{r^{2} f} \sum_{a=1}^{d}\left(\frac{\partial X^{a}}{\partial x^{a}}\right)^{2}, \quad \Lambda_{1,2}=-E \frac{\partial R}{\partial r}, \\
& \Lambda_{1,3}=-\frac{L_{\mathrm{e}}^{2} E}{r^{2} f} \frac{\partial X^{1}}{\partial x^{1}}, \ldots, \Lambda_{1, d+2}=-\frac{L_{\mathrm{e}}^{2} E}{r^{2} f} \frac{\partial X^{d}}{\partial x^{d}}, \\
& \Lambda_{2,1}=E \frac{\partial R}{\partial r}, \quad \Lambda_{2,2}=m^{2} f+E^{2}+\frac{L_{\mathrm{e}}^{2} f}{r^{2}} \sum_{a=1}^{d}\left(\frac{\partial X^{a}}{\partial x^{a}}\right)^{2}, \\
& \Lambda_{2,3}=\frac{L_{\mathrm{e}}^{2} f}{r^{2}} \frac{\partial R}{\partial r} \frac{\partial X^{1}}{\partial x^{1}}, \ldots, \Lambda_{2, d+2}=\frac{L_{\mathrm{e}}^{2} f}{r^{2}} \frac{\partial R}{\partial r} \frac{\partial X^{d}}{\partial x^{d}}, \\
& \Lambda_{3,1}=\frac{L_{\mathrm{e}}^{2} E}{r^{2} f} \frac{\partial X^{1}}{\partial x^{1}}, \quad \Lambda_{3,2}=\frac{L_{\mathrm{e}}^{2} f}{r^{2}} \frac{\partial R}{\partial r} \frac{\partial X^{1}}{\partial x^{1}}, \\
& \Lambda_{3,3}=\frac{m^{2} L_{\mathrm{e}}^{2}}{r^{2}}-\frac{L_{\mathrm{e}}^{4}}{r^{4}} \sum_{a=2}^{d}\left(\frac{\partial X^{a}}{\partial x^{a}}\right)^{2}+\frac{L_{\mathrm{e}}^{2}}{r^{2}}\left(\frac{E^{2}}{f}-f\left(\frac{\partial R}{\partial r}\right)^{2}\right), \\
& \Lambda_{3,4}=\frac{L_{\mathrm{e}}^{4}}{r^{4}} \frac{\partial X^{1}}{\partial x^{1}} \frac{\partial X^{2}}{\partial x^{2}}, \ldots, \Lambda_{3, d+2}=\frac{L_{\mathrm{e}}^{4}}{r^{4}} \frac{\partial X^{1}}{\partial x^{1}} \frac{\partial X^{d}}{\partial x^{d}},
\end{aligned}
$$

$$
\begin{aligned}
\Lambda_{j+2,1} & =\frac{L_{\mathrm{e}}^{2} E}{r^{2} f} \frac{\partial X^{j}}{\partial x^{j}}, \quad \Lambda_{j+2,2}=\frac{L_{\mathrm{e}}^{2} f}{r^{2}} \frac{\partial R}{\partial r} \frac{\partial X^{j}}{\partial x^{j}}, \\
\Lambda_{j+2,3} & =\frac{L_{\mathrm{e}}^{4}}{r^{4}} \frac{\partial X^{j}}{\partial x^{j}} \frac{\partial X^{1}}{\partial x^{1}}, \ldots \Lambda_{j+2, j+1}=\frac{L_{\mathrm{e}}^{4}}{r^{4}} \frac{\partial X^{j}}{\partial x^{j}} \frac{\partial X^{j-1}}{\partial x^{j-1}} \\
\Lambda_{j+2, j+2} & =\frac{m^{2} L_{\mathrm{e}}^{2}}{r^{2}}-\frac{L_{\mathrm{e}}^{4}}{r^{4}} \sum_{a=1, a \neq j}^{d}\left(\frac{\partial X^{a}}{\partial x^{a}}\right)^{2}+\frac{L_{\mathrm{e}}^{2}}{r^{2}}\left(\frac{E^{2}}{f}-f\left(\frac{\partial R}{\partial r}\right)^{2}\right), \\
\Lambda_{j+2, j+3} & =\frac{L_{\mathrm{e}}^{4}}{r^{4}} \frac{\partial X^{j}}{\partial x^{j}} \frac{\partial X^{j+1}}{\partial x^{j+1}}, \ldots, \Lambda_{j+2, d+2}=\frac{L_{\mathrm{e}}^{4}}{r^{4}} \frac{\partial X^{j}}{\partial x^{j}} \frac{\partial X^{d}}{\partial x^{d}} .
\end{aligned}
$$

The condition of the matrix equation to have a non-trivial solution is $\operatorname{det} \Lambda=0$, which will give us a simple expression

$$
\begin{aligned}
& L_{\mathrm{e}}^{4}\left(-\left(\frac{\mathrm{d}}{\partial r} R\right)^{2} f^{2} r^{2}-L_{e}^{2} f \sum_{a=1}^{d}\right. \\
& \left.\times\left(\frac{\partial X^{a}}{\partial x^{a}}\right)^{2}+r^{2}\left(-m^{2} f+E^{2}\right)\right)^{3} m^{2}=0 .
\end{aligned}
$$

It is obvious that the solution to the above equation for the radial function is

$$
R_{ \pm}=\int \mathrm{d} r\left( \pm \frac{1}{f} \sqrt{E^{2}-\frac{L_{e}^{2} f}{r^{2}} \sum_{a=1}^{d}\left(\frac{\partial X^{a}}{\partial x^{a}}\right)^{2}-f m^{2}}\right)
$$

Note that $R_{+}$is for the outgoing massive spin-1 particles while $R_{-}$is for the ingoing ones. It is obvious that there 
is a pole on the event horizon. To solve this singularity in the integral of the imaginary part of $R_{ \pm}$in Eq. (20), we use the residue theorem and complex path integration $[16,17]$. Consequently, the result of the integral is obtained:

$\operatorname{Im} R_{ \pm}= \pm\left.\frac{\pi}{\frac{\mathrm{d}}{\mathrm{d} r} f} E\right|_{r=r_{h}}+k$

where $k$ is a complex integration constant. Then we calculate the tunneling probabilities of the ingoing and outgoing massive spin-1 particles

$$
\begin{aligned}
P_{\text {outgoing }} & =\mathrm{e}^{-\frac{2}{\hbar} \operatorname{Im} S_{+}}=\mathrm{e}^{-2\left(\operatorname{Im} R_{+}+\operatorname{Im} k\right)}, \\
P_{\text {ingoing }} & =\mathrm{e}^{-\frac{2}{\hbar} \operatorname{Im} S_{-}}=\mathrm{e}^{-2\left(\operatorname{Im} R_{-}+\operatorname{Im} k\right)},
\end{aligned}
$$

where we have set $\hbar=1$ in the second equalities. In agreement with the event horizon of the black brane in the GBA theory, particles which are ingoing must be absorbed completely, so that one can choose $P_{\text {ingoing }}=1$. This can be achieved by imposing $\operatorname{Im} k=-\operatorname{Im} R_{-}$. Further recalling $R_{+}=-R_{-}$in (20), the quantum tunneling rate $\Gamma$ of the massive spin-1 particles has the form

$\Gamma=P_{\text {emission }}=\exp \left(-4 \operatorname{Im} R_{+}\right)=\exp \left(-\left.\frac{4 \pi}{\left(\frac{\mathrm{d}}{\mathrm{d} r}\right)} E\right|_{r=r_{h}}\right)$.

We recall from [97] that the equivalent of the tunneling rate $\Gamma$ satisfies the Boltzmann equation $\Gamma=\mathrm{e}^{-\beta E}$ with the Boltzmann factor $\beta$, and the Hawking temperature is defined as $T=\frac{1}{\beta}$. Then we derive the surface temperature of the black brane

$T=\left.\frac{\left(\frac{\mathrm{d}}{\mathrm{d} r} f\right)}{4 \pi}\right|_{r=r_{h}}=\frac{1}{4 \pi}\left((d+1) r_{h}-\frac{L_{e}^{2} \beta^{2}}{2 r_{h}}\right)$.

This agrees well with the Hawking temperature found in Eq. (10) via the Euclidean method.

\section{Hawking temperature via Parikh-Wilczek tunneling approach}

In this section, we use the Parikh-Wilczek Tunneling (PWT) approach to find the Hawking temperature of the black brane solution of Eq. (5). In the PWT approach, one thinks of the tunneling particle as a spherical shell which does not have motion in the $(\theta, \varphi)$-directions. That is to say, we can consider the radial null geodesics of a test particle as a massless spherical shell. With a massless particle, $m=0$, we would presumably say that the particle associated with the wave (photon, or graviton in this case) is massless, and thus they travel along null geodesics. Recently, it is found that there are several phenomena that show the same behavior as quantum tunneling and thus can be accurately described by tunneling. Examples include the tunneling of a classical wave and particle in association [98]. Moreover, the particle feels itself as a barrier, because when the particle tunnels to outside, the radius of the black hole gets smaller depending on the energy of the outgoing particle. Then using the emission and absorption probabilities of the ingoing and the outgoing particles, one can calculate the ratio $[8,9]$

$\Gamma=\frac{P_{\text {emission }}}{P_{\text {absorption }}}=\mathrm{e}^{-\frac{E}{T_{H}}}=\mathrm{e}^{-2 \operatorname{Im} S}$,

where $\operatorname{Im} S$ is for the net imaginary part of action $(\operatorname{Im} S=$ $\operatorname{Im} S_{\text {out }}-\operatorname{Im} S_{\text {in }}$ ). Note that another way to get Eq. (26) is to use the following connections in the WKB limit, which are written in terms of the imaginary part of the action of the particles, $\operatorname{Im} S_{\text {out }}$ and $\operatorname{Im} S_{\text {in }}$ :

$P_{\text {emission }}=\mathrm{e}^{-2 \operatorname{Im} S_{\text {out }}}, \quad P_{\text {absorption }}=\mathrm{e}^{-2 \operatorname{Im} S_{\text {in }}}$.

To proceed, we transform our metric to the non-singular coordinates of Painleve-Gullstrand Coordinates (PGCs) with the transformation [8]

$\mathrm{d} \tau=\mathrm{d} t+\frac{\sqrt{1-f(r)}}{f(r)} \mathrm{d} r$.

It is noted that herein we use $\tau$ as a new time in the PGCs to measure a proper time. Then it is easy to rewrite the metric of Eq. (5) into the form

$\mathrm{d} s^{2}=-f(r) \mathrm{d} \tau^{2}+2 \sqrt{1-f(r)} \mathrm{d} \tau \mathrm{d} r+\mathrm{d} r^{2}+\frac{r^{2}}{L_{e}^{2}} \mathrm{~d} x^{a} \mathrm{~d} x^{a}$.

In the PGCs, the function of the metric is regular, and the singularity is removed at $r=r_{h}$ (i.e., $f=0$ ). We have the only radial null geodesics

$\dot{r}=\frac{\mathrm{d} r}{\mathrm{~d} \tau}= \pm 1-\sqrt{1-f(r)}$.

It is sufficient to calculate the Hawking radiation. The imaginary part of the action for the outgoing waves is given by [35]

$\operatorname{Im} S_{\text {out }}=\operatorname{Im} \int_{r_{\text {in }}}^{r_{\text {out }}} p_{r} \mathrm{~d} r=\operatorname{Im} \int_{r_{\text {in }}}^{r_{\text {out }}} \int_{0}^{p_{r}} \mathrm{~d} p_{r}^{\prime} \mathrm{d} r$,

where $p_{r}$ is the canonical momentum, $r_{\text {in }}$ and $r_{\text {out }}$ are the initial and final radius of the black hole. The particle tunnels 
through between these radii so the potential barrier is located between these radii.

The total mass of the system $(M)$ is fixed inasmuch as the black hole shrinks ( $r_{\text {in }}>r_{\text {out }}$ ) after the Hawking radiation, so that the black hole can fluctuate. Moreover, in this study, we use only chargeless particles which have a thinspherical-shell of energy $\omega$. Then we consider the decreasing of the mass of the black hole $M \rightarrow M-\omega$, because of the effect of the self-gravitation. Using the Hamilton equation, the momentum is transferred to the energy $\dot{r}=\frac{\mathrm{d} H}{\mathrm{~d} p_{r}}$, and Eq. (31) reduces to

$\operatorname{Im} S_{\text {out }}=\operatorname{Im} \int_{r_{\text {in }}}^{r_{\text {out }}} \int_{M}^{M-\omega} \frac{\mathrm{d} r}{\dot{r}} \mathrm{~d} H$.

One can now use the energy of the particle $\omega$ instead of $H$ to transform $H=M-\omega^{\prime}$ to $\mathrm{d} H=-\mathrm{d} \omega^{\prime}$ as

$\operatorname{Im} S_{\text {out }}=\int_{r_{\text {in }}}^{r_{\text {out }}} \int_{0}^{\omega} \frac{\mathrm{d} r}{\dot{r}}\left(-\mathrm{d} \omega^{\prime}\right)=-\omega \int_{r_{\text {in }}}^{r_{\text {out }}} \frac{\mathrm{d} r}{1-\sqrt{1-f(r)}}$.

We used Eq. (30) in the second equality. To solve the above integral, we use the contour integral by ensuring that positive energy solutions decay in time because there is a pole at the horizon where $\dot{r}=0$. For the tunneling particle, we obtain the imaginary part of the action,

$\operatorname{Im} S_{\text {out }}=\frac{2 \pi \omega}{f^{\prime}\left(r_{h}\right)}+O\left(\omega^{2}\right)$.

On the other hand, the ingoing particle can be ignored, because the probability amplitude of the ingoing particle is unity so we use the above tunneling rate for an outgoing particle from the horizon. Then we find that the tunneling rate is

$\Gamma=\exp \left(-4 \pi \omega / f^{\prime}\left(r_{h}\right)\right)$

and the Hawking temperature is

$T=\left.\frac{\left(\frac{\mathrm{d}}{\mathrm{d} r} f\right)}{4 \pi}\right|_{r=r_{h}}=\frac{1}{4 \pi}\left((d+1) r_{h}-\frac{L_{e}^{2} \beta^{2}}{2 r_{h}}\right)$.

This expression of the Hawking temperature is the same as the Eqs. (25) and (10). Hence, we have correctly recovered the Hawking temperature.

\section{Conclusion}

In this paper, we have studied the tunneling of spin-1 particles from arbitrary dimensional AdS black branes with GB corrections and additional axion fields, using the HamiltonJacobi approach to recover the corresponding Hawking temperature. And then we have checked our results using the
Parikh-Wilczek approach. Firstly, to investigate the tunneling rate of the spin-1 particles, we have derived the solutions of the Proca equation on the background of the $d+2$ dimensional black brane in Gauss-Bonnet-Axions theory with the help of Hamilton-Jacobi approach. To solve these solutions, we have used the separation of variables and extracted the radial part of the wave solution after taking the determinant of the matrix zero. Then we have obtained the solution of the complex integral on the event horizon using the residue method. The tunneling rate of the spin-1 particles has been calculated and the Hawking temperature is correctly confirmed compared with the Boltzmann factor. Secondly, we have used the Parikh-Wilczek approach which involves the null geodesic equation of the emitted scalar particles for the derivation of the Hawking temperature.

Remarkably, we have showed that the radiation spectra are not purely thermal. There is an effect of the Gauss-Bonnet term on the radiation spectra that means that the information can be extracted using this term. This is an important result because it is well known that GB parameters have no effect on the temperature of GB black branes with planar topology [83-85]. However, in this model of the GBA gravitational theory [79], it is clear that the GB coupling term affects the Hawking temperature as well as the information paradox. One can store all information of the black brane inside the GB term to make it survive from the loss of information through tunneling from the event horizon. It is noted that if one removes the axions field, the temperature of the normal GB theory is recovered [79]. We can conclude that the magnitude of the Hawking temperature depends on the values of the parameters of GB. It is worth to mention that we have neglected the back-reaction effects due to the radiation of particles and also self-gravitating effects. We have calculated the Hawking temperature only by a leading term. This work shows that the gravitational theories with higher curvature coupling, such as GB corrections and the higher dimensions are significantly influential.

Lastly, from Einstein's theory of gravity one concludes that black holes have no hair. Recently, using the data of gravitational waves from the LIGO, information of the hair of the black hole is successfully extracted [99]. In future, this kind of experiments may also shed light on the extraction of information from black holes/branes and solve the information paradox.

Acknowledgements This work is partly supported by Chilean FONDECYT grant No.3150006 (X.M. Kuang) and No.3170035 (A. Övgün).

Open Access This article is distributed under the terms of the Creative Commons Attribution 4.0 International License (http://creativecomm ons.org/licenses/by/4.0/), which permits unrestricted use, distribution, and reproduction in any medium, provided you give appropriate credit to the original author(s) and the source, provide a link to the Creative Commons license, and indicate if changes were made.

Funded by $\mathrm{SCOAP}^{3}$. 


\section{References}

1. S.W. Hawking, Nature (Lond.) 248, 30 (1974)

2. S.W. Hawking, Commun. Math. Phys. 43, 199 (1975) [46, 206 (1976), Erratum]

3. S.W. Hawking, M.J. Perry, A. Strominger, Phys. Rev. Lett. 116, 231301 (2016)

4. S.W. Hawking, M.J. Perry, A. Strominger, JHEP 1705, 161 (2017)

5. A. Strominger. arXiv: 1706.07143 [hep-th]

6. W.G. Unruh, Phys. Rev. D 14, 870 (1976)

7. T. Damour, R. Ruffini, Phys. Rev. D 14, 332 (1976)

8. M.K. Parikh, F. Wilczek, Phys. Rev. Lett. 85, 5042 (2000)

9. M.K. Parikh, Int. J. Mod. Phys. D 13, 2351 (2004)

10. K. Srinivasan, T. Padmanabhan, Phys. Rev. D 60, 024007 (1999)

11. I. Sakalli, Int. J. Theor. Phys. 50, 2426 (2011)

12. G. Clément, J.C. Fabris, G.T. Marques, Phys. Lett. B 651, 54 (2007)

13. J. Zhang, Z. Zhao, JHEP 10, 055 (2005)

14. J. Zhang, Z. Zhao, Phys. Lett. B 618, 14 (2005)

15. J. Zhang, Z. Zhao, Phys. Lett. B 638, 110 (2006)

16. M. Angheben, M. Nadalini, L. Vanzo, S. Zerbini, J. High Energy Phys. 05, 014 (2005)

17. M. Angheben, M. Nadalini, L. Vanzo, S. Zerbini, J. High Energy Phys. 05, 037 (2005)

18. R. Kerner, R.B. Mann, Phys. Rev. D 73, 104010 (2006)

19. R. Kerner, R.B. Mann, Class. Quantum Grav. 25, 095014 (2008)

20. R. Kerner, R.B. Mann, Phys. Lett. B 665, 277 (2008)

21. R. Li, J.-R. Ren, Phys. Lett. B 661, 370 (2008)

22. R. Li, J.-R. Ren, S.-W. Wei, Class. Quantum Grav. 25, 125016 (2008)

23. Ran Li, Junkun Zhao, Eur. Phys. J. Plus 131, 249 (2016)

24. D.-Y. Chen, Q.-Q. Jiang, X.-T. Zu, Phys. Lett. B 665, 106 (2008)

25. Q.-Q. Jiang, Phys. Rev. D 78, 044009 (2008)

26. A. Yale, R.B. Mann, Phys. Lett. B 673, 168 (2009)

27. D. Chen, H. Wu, H. Yang, Adv. High Energy Phys. 2013, 432412 (2013)

28. S.I. Kruglov, Mod. Phys. Lett. A 29, 1450203 (2014)

29. S.I. Kruglov, Int. J. Mod. Phys. A 29, 1450118 (2014)

30. G.-R. Chen, S. Zhou, Y.-C. Huang, Int. J. Mod. Phys. D 24, 1550005 (2015)

31. I. Sakalli, A. Ovgun, Eur. Phys. J. Plus 130, 110 (2015)

32. X.-Q. Li, G.-R. Chen, Phys. Lett. B 751, 34-38 (2015)

33. GuQiang Li, J. Phys. A Math. Gen. 39, 11889 (2006)

34. Z. Feng, Y. Chen, X. Zu, Astrophys. Space Sci. 359, 48 (2015)

35. Gu-Qiang Li, Jie-Xiong Mo, Gen. Rel. Grav. 49(4), 57 (2017)

36. R. Becar, P. Gonzalez, G. Pulgar, Joel Saavedra, Int. J. Mod. Phys. A 25, 1463 (2010)

37. I. Sakalli, A. Ovgun, EPL 110, 10008 (2015)

38. E.T. Akhmedov, V. Akhmedova, D. Singleton, Phys. Lett. B 642, 124 (2006)

39. E.T. Akhmedov, V. Akhmedova, T. Pilling, D. Singleton, Int. J. Mod. Phys. A 22, 1705 (2007)

40. V. Akhmedova, T. Pilling, A. de Gill, D. Singleton, Phys. Lett. B 666, 269 (2008)

41. X.X. Zeng, J.S. Hou, S.Z. Yang, Pramana 70, 409 (2008)

42. X.X. Zeng, S.Z. Yang, Gen. Rel. Grav. 40, 2107 (2008)

43. B.R. Majhi, Phys. Rev. D 79, 044005 (2009)

44. H. Pasaoglu, I. Sakalli, Int. J. Theor. Phys. 48, 3517 (2009)

45. I. Sakalli, M. Halilsoy, H. Pasaoglu, Int. J. Theor. Phys. 50, 3212 (2011)

46. A. Övgün, Int. J. Theor. Phys. 55(6), 2919-2927 (2016)

47. I. Sakalli, M. Halilsoy, H. Pasaoglu, Astrophys. Space Sci. 340, 155 (2012)

48. I. Sakalli, M. Halilsoy, H. Pasaoglu, Astrophys. Space Sci. 357, 95 (2015) (Erratum)

49. I. Sakalli, A. Ovgun, Astrophys. Space Sci. 359, 32 (2015)
50. I. Sakalli, A. Övgün, K. Jusufi, Astrophys. Space Sci. 361(10), 330 (2016)

51. R. da Rocha, J.M. Hoff da Silva, EPL 107, 50001 (2014)

52. J. Ahmed, K. Saifullah, JCAP 08, 011 (2011)

53. I. Sakalli, H. Gursel, Eur. Phys. J. C 76, 318 (2016)

54. I. Sakalli, A. Ovgun, Eur. Phys. J. Plus 130, 110 (2015)

55. I. Sakalli, A. Ovgun, Gen. Relativ. Gravit. 48, 1 (2016)

56. A. Ovgün, K. Jusufi, Eur. Phys. J. Plus 131, 177 (2016)

57. K. Jusufi, A. Ovgun, Astrophys. Space Sci. 361, 207 (2016)

58. K. Jusufi, A. Ovgun, G. Apostolovska, Adv. High Energy Phys. 2017, 7 (2017). Article id 8798657

59. K. Jusufi, A. Ovgun, Int. J. Theor. Phys. 56(6), 1725-1738 (2017)

60. K. Jusufi, Europhys. Lett. 116(6), 60013 (2016)

61. K. Jusufi, Gen. Rel. Grav., 48(8), 105 (2016)

62. K. Jusufi, Gen. Rel. Gravit. 47(10), 124 (2015)

63. S. Shankaranarayanan, K. Srinivasan, T. Padmanabhan, Mod. Phys. Lett. A 16, 571 (2001)

64. S. Haldar, C. Corda, S. Chakraborty. arXiv:1705.08307 [gr-qc]

65. A. Biswas. arXiv: 1705.01116 [hep-th]

66. W. Javed, R. Ali, G. Abbas. arXiv:1705.05702 [physics.gen-ph]

67. M. Hossain Ali, K. Sultana, Int. J. Theor. Phys. 56(7), 2279-2292 (2017)

68. Z. Feng, S. Yang. arXiv:1706.01598 [gr-qc]

69. M. Hossain Ali, Class. Quant. Grav. 24, 5849-5860 (2007)

70. M. Hossain Ali, Int. J. Theor. Phys. 47, 2203-2217 (2008)

71. M. Hossain Ali, Gen. Rel. Gravit. 36, 1171-1181 (2004)

72. H.M. Siahaan, Eur. Phys. J. C 76(3), 139 (2016)

73. J.C. Martinez, E. Polatdemir, Eur. Phys. J. C 21, 389-395 (2001)

74. Tao Zhu, Ji-Rong Ren, Eur. Phys. J. C 62, 413-418 (2009)

75. G. Blado, T. Prescott, J. Jennings, J. Ceyanes, R. Sepulveda, Eur. J. Phys. 37, 025401 (2016)

76. G. Gecim, Y. Sucu, Eur. Phys. J. Plus 132(3), 105 (2017)

77. G. Gecim, Y. Sucu, JCAP 1302, 023 (2013)

78. J. Ahmed, K. Saifullah, JCAP 1111, 023 (2011)

79. X.M. Kuang, J.P. Wu, Phys. Lett. B 770, 117 (2017)

80. J. Beringer et al., Particle Data Group, Phys. Rev. D. 86, 010001 (2012)

81. ATLAS Collaboration (G. Aad et al.), Phys. Lett. B 716, 1 (2012)

82. T. Andrade, B. Withers, JHEP 1405, 101 (2014)

83. D.G. Boulware, S. Deser, Phys. Rev. Lett. 55, 2656 (1985)

84. R.-G. Cai, Phys. Rev. D 65, 084014 (2002)

85. M. Cvetic, S. Nojiri, S.D. Odintsov, Nucl. Phys. B 628, 295 (2002)

86. L. Cheng, X.H. Ge, Z.Y. Sun, JHEP 1504, 135 (2015)

87. A. Buchel, J. Escobedo, R.C. Myers, M.F. Paulos, A. Sinha, M. Smolkin, JHEP 1003, 111 (2010)

88. M. Brigante, H. Liu, R.C. Myers, S. Shenker, S. Yaida, Phys. Rev. Lett. 100, 191601 (2008)

89. X.O. Camanho, J.D. Edelstein, JHEP 1004, 007 (2010)

90. X.H. Ge, S.J. Sin, JHEP 0905, 051 (2009)

91. X.O. Camanho, J.D. Edelstein, J. Maldacena, A. Zhiboedov, JHEP 1602, 020 (2016)

92. G. D'Appollonio, P. Di Vecchia, R. Russo, G. Veneziano, JHEP 1505, 144 (2015)

93. R.A. Konoplya, A. Zhidenko, Phys. Rev. D 95(10), 104005 (2017)

94. R.A. Konoplya, A. Zhidenko, JCAP 1705(05), 050 (2017)

95. R.A. Konoplya, A. Zhidenko. arXiv:1705.07732 [hep-th]

96. Y.L. Wang, X.H. Ge, Phys. Rev. D 94(6), 066007 (2016)

97. L. Vanzo, G. Acquaviva, R. Di Criscienzo, Class. Quantum Gravit. 28, 18 (2011)

98. A. Eddi, E. Fort, F. Moisy, Y. Couder, Phys. Rev. Lett. 102, 240401 (2009)

99. H. Yang, K. Yagi, J. Blackman, L. Lehner, V. Paschalidis, F. Pretorius, N. Yunes, Phys. Rev. Lett. 118, 161101 (2017) 